This is a self-archived - parallel published version of this article in the publication archive of the University of Vaasa. It might differ from the original.

\title{
The Valuation of European Option Under Subdiffusive Fractional Brownian Motion of the Short Rate
}

\author{
Author(s): Shokrollahi, Foad
}

Title: $\quad$ The Valuation of European Option Under Subdiffusive Fractional Brownian Motion of the Short Rate

Year: $\quad 2020$

Version: Accepted manuscript

Copyright (C) World Scientific Publishing Company, https://www.worldscientific.com/worldscinet/ijtaf. Electronic version of an article published as International Journal of Theoretical and Applied Finance 23(4), 1-16.

https://doi.org/10.1142/So219024920500223

Please cite the original version:

Shokrollahi, F. (2020). The Valuation of European Option Under Subdiffusive Fractional Brownian Motion of the Short Rate.

International Journal of Theoretical and Applied Finance 23(4), 1-16. https://doi.org/10.1142/So219024920500223 


\title{
THE VALUATION OF EUROPEAN OPTION UNDER SUBDIFFUSIVE FRACTIONAL BROWNIAN MOTION MECHANISM OF THE SHORT RATE
}

\author{
FOAD SHOKROLLAHI \\ Department of Mathematics and Statistics, University of Vaasa, P.O. Box 700, \\ FIN-65101 Vaasa, FINLAND
}

\begin{abstract}
In this paper we propose an extension of the Merton model. We apply the subdiffusive mechanism to analyze European option in a fractional Black-Scholes environment, when the short rate follows the subdiffusive fractional Black-Scholes model. We derive a pricing formula for call and put options and discuss the corresponding fractional Black-Scholes equation. We present some features of our model pricing model for the cases of $\alpha$ and $H$.
\end{abstract}

\section{INTRODUCTION}

The pioneer study of the option pricing was introduced by Black-Scholes [1] in 1973. In the Black-Scholes $(B S)$ model has been assumed that the underlying assets follows a geometric Broawinian motion. While, there exist a series of evidence which show the $B S$ model unable to cover substantial behavior from financial markets such as: long-range dependence, heavy-tailed and periods of constant values. Hence, they proposed various modifications of the $B S$ model to capture these shortcomings.

One of well developed modifications of the $B S$ model is the fractional BlackScholes model which, describes long-range dependence and self-similarity from financial data. In the fractional Black-Scholes $(F B S)$ model, the Brownian motion is substituted with the fractional Brownian motion $(F B M)$ in the $B S$ model. For more details about fractional Black-Scholes model, you can see [16, 14, 2, 13].

Furthermore, analysis of financial data displays that various processes viewed in finance show special terms in which they are constant [8]. The same property is observed in physical system with subduffusion. The fixed terms of financial processes according to the trapping event in which the subdiffusive examination particle is constant [4]. The mathematical interpretation of subdiffusion is in terms of Fractional Fokker Planck equation $(F F P E)$. This equation was introduced from the continuous time random walk strategy with fat tail waiting times [12], later used as a substantial tool to evaluate complex system with slow dynamics. In this paper, we use the $F B S$ model in subdiffusive mechanism to better describe

E-mail address: foad.shokrollahi@uva.fi.

Date: April 15, 2019.

2010 Mathematics Subject Classification. 91G20; 91G80; 60G22.

Key words and phrases. Merton short rate model; Subdiffusive processes; Fractional Brownian motion; Option pricing. 
behaviour from financial markets. We use the same strategy in [11, 15], which the objective time $t$ is replaced by the inverse $\alpha$-stable subordinator $T_{\alpha}(t)$ in the $F B S$ model. Then, the dynamic of asset price is given by the following subdiffusive $F B S$

$$
\begin{aligned}
d S_{\alpha}(t) & = \\
d S\left(T_{\alpha}(t)\right) & =\mu_{s} S\left(T_{\alpha}(t)\right) d\left(T_{\alpha}(t)\right)+\sigma_{s} S\left(T_{\alpha}(t)\right) d B_{1}^{H}\left(T_{\alpha}(t)\right),
\end{aligned}
$$

where $\mu_{s}, \sigma_{s}$ are constant, $B_{1}^{H}$ is $F B M$ with Hurst parameter $H \in\left[\frac{1}{2}, 1\right)$. $T_{\alpha}(t)$ is the inverse $\alpha$-stable subordinator with $\alpha \in(0,1)$ defined as follows

$$
T_{\alpha}(t)=\inf \left\{\tau>0: U_{\alpha}(\tau)>t\right\},
$$

$T_{\alpha}(t)$ is assumed to be independent of $B_{1}^{H} . \quad\left\{U_{\alpha}(t)\right\}_{t \geq 0}$ is a $\alpha$-stable Levy process with nonnegative increments and Laplace transform: $E\left(e^{-u U_{\alpha}(t)}\right)=e^{-t u^{\alpha}}$ $[5,17,7]$. when $\alpha \uparrow 1$, the $T_{\alpha}(t)$ degenerates to $t$.

On the other hand, all above studies have assumed that the short rate is constant during the life of an option. However, in reality the short rate is evolving randomly over time. Hence, in order to take into account the stochastic short rate, we assume that the short rate $r(t)=\hat{S}\left(T_{\alpha}(t)\right)$ follows:

$$
\begin{aligned}
d \hat{S}_{\alpha}(t) & = \\
d \hat{S}\left(T_{\alpha}(t)\right) & =\mu_{r} d T_{\alpha}(t)+\sigma_{r} d B_{2}^{H}\left(T_{\alpha}(t)\right),
\end{aligned}
$$

here $\mu_{r}, \sigma_{r}$ are constant, $B_{2}^{H}$ is $F B M$ with Hurst parameter $H \in\left[\frac{1}{2}, 1\right)$ and $T_{\alpha}(t)$ is assumed to be independent of $B_{2}^{H}$.
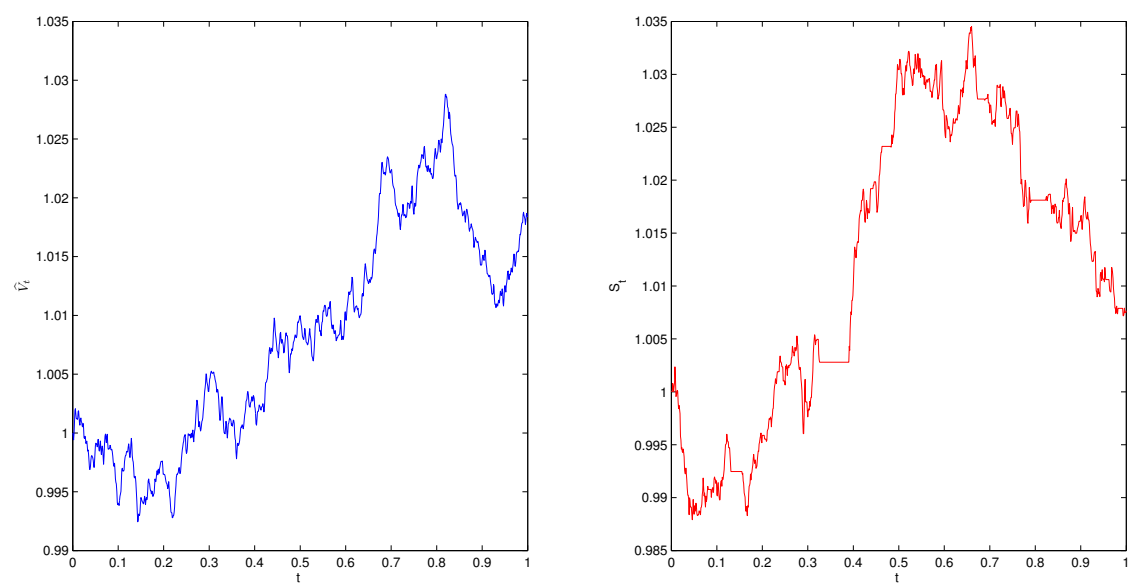

FigURE 1. discrepancy and relation between the sample paths of the stock price in the $F B S$ model (left) and the subdiffusive $F B S$ model (right) for $r=0.01, \alpha=0.9, H=0.8, \sigma=0.1, S_{0}=1$.

The first contribution of this paper is to propose a valuation model to price a zero-coupon bond by applying the subdiffusive mechanism of the short rate. The 
second contribution is to value an European option when the asset price and short rate are follow subdiffusive $F B S$ model. This paper is organized as follows. In the next section, we derive a new model to value a riskless zero-coupon bond paying $\$ 1$ at maturity. In Section 3, we obtain the corresponding FBS equation by using delta hedging argument and discuss some special cases of this equation. In Section 4 , we propose a pricing model for the European call and put options. Some particular features and simulation studies of our sudiffusive model are discussed in Section 5. Section 6 concludes this research.

\section{PRICING MODEL FOR A ZERO-COUPON BOND}

We assume that the short rate $r(t)$ satisfy Equation (1.3), $\alpha \in\left(\frac{1}{2}, 1\right)$ and $2 \alpha-$ $\alpha H>1$, then by using the Taylor series expansion to $P(r, t, T)$, we obtain

$$
\begin{aligned}
P(r+\Delta r, t+\Delta t)= & P(r, t, T)+\frac{\partial P}{\partial r} \Delta r+\frac{\partial P}{\partial t} \Delta t \\
(2.1) \quad & +\frac{1}{2} \frac{\partial^{2} P}{\partial r^{2}}(\Delta r)^{2}++\frac{1}{2} \frac{\partial^{2} P}{\partial r \partial t} \Delta r(\Delta t)+\frac{1}{2} \frac{\partial^{2} P}{\partial t^{2}}(\Delta t)^{2}+O(\Delta t) .
\end{aligned}
$$

From, Equation (1.3) and [17], we have

$$
\begin{aligned}
\Delta r & =\mu_{r}\left(\Delta T_{\alpha}(t)\right)+\sigma_{r} B_{1}^{H}\left(T_{\alpha}(t)\right) \\
& =\mu_{r}\left(\frac{t^{\alpha-1}}{\Gamma(\alpha)}\right)^{2 H}(\Delta t)^{2 H}+\sigma_{r} \Delta B_{1}^{H}\left(T_{\alpha}(t)\right)+O\left((\Delta t)^{2 H}\right) . \\
(\Delta r)^{2} & =\sigma_{r}^{2}\left(\frac{t^{\alpha-1}}{\Gamma(\alpha)}\right)^{2 H}(\Delta t)^{2 H}+O\left((\Delta t)^{2 H}\right) . \\
\Delta r(\Delta t) & =O\left((\Delta t)^{2 H}\right) .
\end{aligned}
$$

Then from the Lemma 1 in [17], we can get

$$
\begin{aligned}
d P(r, t, T)= & {\left[\left(\frac{t^{\alpha-1}}{\Gamma(\alpha)}\right)^{2 H}\left(\mu_{r} \frac{\partial P}{\partial r}+\frac{1}{2} \sigma_{r}^{2} \frac{\partial^{2} P}{\partial r^{2}}\right) 2 H t^{2 H-1}+\frac{\partial P}{\partial t}\right] d t } \\
& +\sigma_{r} \frac{\partial P}{\partial t} d B_{1}^{H}\left(T_{\alpha}(t)\right) .
\end{aligned}
$$

Assuming

$$
\begin{aligned}
\mu & =\frac{1}{P}\left[\left(\frac{t^{\alpha-1}}{\Gamma(\alpha)}\right)^{2 H}\left(\mu_{r} \frac{\partial P}{\partial r}+\frac{1}{2} \sigma_{r}^{2} \frac{\partial^{2} P}{\partial r^{2}}\right) 2 H t^{2 H-1}+\frac{\partial P}{\partial t}\right] \\
\sigma & =\frac{1}{P}\left(\frac{\partial P}{\partial r}\right)
\end{aligned}
$$


and letting the local expectations hypothesis holds for the term structure of interest rates (i.e. $\mu=r$ ), we have

$$
\begin{aligned}
& \frac{\partial P}{\partial t}+2 H t^{2 H-1} \mu_{r}\left(\frac{t^{\alpha-1}}{\Gamma(\alpha)}\right)^{2 H} \frac{\partial P}{\partial r} \\
& +H t^{2 H-1} \sigma_{r}^{2}\left(\frac{t^{\alpha-1}}{\Gamma(\alpha)}\right)^{2 H} \frac{\partial^{2} P}{\partial r^{2}}-r P=0 .
\end{aligned}
$$

Then, zero-coupon bond $P(r, t, T)$ with boundary condition $P(r, t, T)=1$ satisfy the following partial differential equation

$$
\begin{aligned}
& \frac{\partial P}{\partial t}+2 H t^{2 H-1} \mu_{r}\left(\frac{t^{\alpha-1}}{\Gamma(\alpha)}\right)^{2 H} \frac{\partial P}{\partial r} \\
& +H t^{2 H-1} \sigma_{r}^{2}\left(\frac{t^{\alpha-1}}{\Gamma(\alpha)}\right)^{2 H} \frac{\partial^{2} P}{\partial r^{2}}-r P=0 .
\end{aligned}
$$

To solve Equation (2.8) for $P(r, t, T)$, let $\tau=T-t, P(r, t, T)=\exp \left\{f_{1}(\tau)-\right.$ $\left.r f_{2}(\tau)\right\}$, then we can get

$$
\begin{aligned}
& \frac{\partial P}{\partial t}= P\left(-\frac{\partial f_{1}(\tau)}{\partial t}+r \frac{\partial f_{2}(\tau)}{\partial t}\right), \\
& \frac{\partial P}{\partial r}=-P f_{2}(\tau), \\
& \frac{\partial^{2} P}{\partial r^{2}}=P f_{2}(\tau)^{2} .
\end{aligned}
$$

Replacing Equations (2.10) and (2.11) into Equation (2.9) and simplifying Equation (2.8) becomes

$$
\begin{aligned}
& P\left[H t^{2 H-1} \sigma_{r}^{2} f_{2}(\tau)^{2}\left(\frac{t^{\alpha-1}}{\Gamma(\alpha)}\right)^{2 H}-2 H t^{2 H-1} \mu_{r} f_{2}(\tau)\left(\frac{t^{\alpha-1}}{\Gamma(\alpha)}\right)^{2 H}\right. \\
& \left.-\frac{\partial f_{1}(\tau)}{\partial \tau}+r\left(\frac{\partial f_{2}(\tau)}{\partial t}-1\right)\right]=0 .
\end{aligned}
$$

From Equation (2.12), we obtain

$$
\begin{aligned}
& \frac{\partial f_{1}(\tau)}{\partial \tau}=H t^{2 H-1}\left(\frac{t^{\alpha-1}}{\Gamma(\alpha)}\right)^{2 H}\left(\sigma_{r}^{2} f_{2}(\tau)^{2}-2 \mu_{r} f_{2}(\tau)\right), \\
& \frac{\partial f_{2}(\tau)}{\partial \tau}=1 .
\end{aligned}
$$

Then,

$$
\begin{aligned}
f_{1}(\tau)= & \frac{H \sigma_{r}^{2}}{(\Gamma(\alpha))^{2 H}} \int_{0}^{\tau}(T-s)^{(\alpha-1) 2 H+2 H-1} s^{2} d s \\
& -\frac{2 H \mu_{r}}{(\Gamma(\alpha))^{2 H}} \int_{0}^{\tau}(T-s)^{(\alpha-1) 2 H+2 H-1} s d s \\
f_{2}(\tau)= & \tau .
\end{aligned}
$$

Therefore, we derive a pricing model for a riskless zero-coupon bond.

$$
P(r, t, T)=e^{-r \tau+f_{1}(\tau)} .
$$


Corollary 2.1. When $\alpha \uparrow 1$, Equations (1.3) and (1.1) reduce to the FBM, we obtain

$$
f_{1}(\tau)=H \sigma_{r}^{2} \int_{0}^{\tau}(T-s)^{2 H-1} s^{2} d s-2 H \mu_{r} \int_{0}^{\tau}(T-s)^{2 H-1} s d s,
$$

specially, if $t=0$

$$
f_{1}(\tau)=\sigma_{r}^{2} \frac{T^{2 H+2}}{(2 H+1)(2 H+2)}-\mu_{r} \frac{T^{2 H+1}}{2 H+1}
$$

then

$$
P(r, t, T)=\exp \left\{-r T+\sigma_{r}^{2} \frac{T^{2 H+2}}{(2 H+1)(2 H+2)}-\mu_{r} \frac{T^{2 H+1}}{2 H+1}\right\}
$$

Corollary 2.2. If $H=\frac{1}{2}$, from Equation (2.14), we obtain

$$
\begin{aligned}
f_{1}(\tau)= & \frac{1}{2} \frac{\sigma_{r}^{2}}{\Gamma(\alpha)} \int_{0}^{\tau}(T-s)^{\alpha-1} s^{2} d s \\
& -\frac{\mu_{r}}{\Gamma(\alpha)} \int_{0}^{\tau}(T-s)^{\alpha-1} s d s
\end{aligned}
$$

then the result is consistent with the result in [6].

Further, if $\alpha \uparrow 1$ and $H=\frac{1}{2}$, Equations (1.3) and (1.1) reduce to the geometric Brownian motion, then we have

$$
f_{1}(\tau)=\frac{1}{6} \sigma_{r}^{2} \tau^{3}-\frac{1}{2} \mu_{r} \tau^{2}
$$

then

$$
P(r, t, T)=e^{-r \tau+\frac{1}{6} \sigma_{r}^{2} \tau^{3}-\frac{1}{2} \mu_{r} \tau^{2}} .
$$

which is consistent with the result in $[9,3]$.

\section{Fractional Black-Scholes equation}

This section provides corresponding $F B S$ equation for European options when the short rate and stock price satisfy Equations (1.3) and (1.1), respectively, here $B_{1}^{H}$ and $B_{2}^{H}$ are two dependent $F B M$ with Hurst parameter $H \in\left[\frac{1}{2}, 1\right)$ and correlation coefficient $\rho$.

Let $C=C(S, r, t)$ be the price of a European call option at time $t$ with a strike price $K$ that matures at time $T$. Then we have.

Theorem 3.1. Assume that the short rate $r(t)$ and stock price $S(t)$ satisfy Equations (1.3) and (1.1), respectively. Then, $C(S, r, t)$ is the solution the following equation:

$$
\begin{aligned}
& \frac{\partial C}{\partial t}+\widetilde{\sigma}_{s}^{2}(t) S^{2} \frac{\partial^{2} C}{\partial S^{2}}+\widetilde{\sigma}_{r}^{2}(t) \frac{\partial^{2} C}{\partial r^{2}}+2 \rho \widetilde{\sigma}_{r}(t) \widetilde{\sigma}_{s}(t) \frac{\partial^{2} C}{\partial S \partial r} \\
& +2 H t^{2 H-1} \mu_{r}\left(\frac{t^{\alpha-1}}{\Gamma(\alpha)}\right)^{2 H} \frac{\partial C}{\partial r}+r S \frac{\partial C}{\partial S}-r C=0
\end{aligned}
$$


where

$$
\begin{aligned}
& \tilde{\sigma}_{s}^{2}(t)=H t^{2 H-1} \sigma_{s}^{2}\left(\frac{t^{\alpha-1}}{\Gamma(\alpha)}\right)^{2 H}, \\
& \widetilde{\sigma}_{r}^{2}(t)=H t^{2 H-1} \sigma_{r}^{2}\left(\frac{t^{\alpha-1}}{\Gamma(\alpha)}\right)^{2 H} .
\end{aligned}
$$

$\sigma_{s}, \sigma_{r}, \mu_{s}, \mu_{s}$, are constant, $H \in\left[\frac{1}{2}, 1\right)$ and $\alpha \in\left(\frac{1}{2}, 1\right)$ and $2 \alpha-\alpha H>1$.

Proof: We consider a portfolio with $D_{1 t}$ units of stock and $D_{2 t}$ units of zerocoupon bond $P(r, t, T)$ and one unit of $C=C(r, t, T)$. Then, the value of the portfolio at current time $t$ is

$$
\Pi_{t}=C-D_{1 t} S_{t}-D_{2 t} P_{t} .
$$

Then, from [6] we have

$$
\begin{aligned}
d \Pi_{t} & =C_{t}-D_{1 t} d S_{t}-D_{2 t} d P_{t} \\
& =\left[\frac{\partial C}{\partial t} d t+H t^{2 H-1} \sigma_{s}^{2} S_{t}^{2}\left(\frac{t^{\alpha-1}}{\Gamma(\alpha)}\right)^{2 H} \frac{\partial^{2} C}{\partial S^{2}}+H t^{2 H-1} \sigma_{r}^{2}\left(\frac{t^{\alpha-1}}{\Gamma(\alpha)}\right)^{2 H} \frac{\partial^{2} C}{\partial r^{2}}\right. \\
& \left.+2 H t^{2 H-1} \rho \sigma_{r} \sigma_{s} S\left(\frac{t^{\alpha-1}}{\Gamma(\alpha)}\right)^{2 H} \frac{\partial^{2} C}{\partial S \partial r}\right] d t+\left[\frac{\partial C}{\partial t}-D_{1 t}\right] d S_{t} \\
& +\left[\frac{\partial C}{\partial r}-D_{2 t} \frac{\partial P}{\partial r}\right] d r+D_{2 t}\left[\frac{\partial P}{\partial t}+H t^{2 H-1} \sigma_{r}^{2}\left(\frac{t^{\alpha-1}}{\Gamma(\alpha)}\right)^{2 H} \frac{\partial^{2} P}{\partial r^{2}}\right] d t .
\end{aligned}
$$

By setting $D_{1 t}=\frac{\partial C}{\partial S}, D_{2 t}=\frac{\frac{\partial C}{\partial r}}{\frac{\partial P}{\partial r}}$, to eliminate the stochastic noise, then

$$
\begin{aligned}
d \Pi_{t} & =\left[\frac{\partial C}{\partial t}+H t^{2 H-1}\left(\frac{t^{\alpha-1}}{\Gamma(\alpha)}\right)^{2 H}\left(\sigma_{s}^{2} S^{2} \frac{\partial^{2} C}{\partial S^{2}}+\sigma_{r}^{2} \frac{\partial^{2} C}{\partial r^{2}}+2 \rho \sigma_{r} \sigma_{s} S \frac{\partial^{2} C}{\partial S \partial r}\right)\right] d t \\
& =\frac{\partial C}{\partial r}\left[r P-2 H t^{2 H-1} \mu_{r}\left(\frac{t^{\alpha-1}}{\Gamma(\alpha)}\right)^{2 H} \frac{\partial P}{\partial r}\right] d t .
\end{aligned}
$$

The return of an amount $\Pi_{t}$ invested in bank account is equal to $r(t) \Pi_{t} d t$ at time $d t, \mathbb{E}\left(d \Pi_{t}\right)=r(t) \Pi_{t} d t=r(t)\left(C-D_{1 t} S_{t}-D_{2 t} P_{t}\right)$, hence from Equation (3.6) we have

$$
\begin{aligned}
& \frac{\partial C}{\partial t}+H t^{2 H-1}\left(\frac{t^{\alpha-1}}{\Gamma(\alpha)}\right)^{2 H}\left(\sigma_{s}^{2} S^{2} \frac{\partial^{2} C}{\partial S^{2}}+\sigma_{r}^{2} \frac{\partial^{2} C}{\partial r^{2}}+2 \rho \sigma_{r} \sigma_{s} S \frac{\partial^{2} C}{\partial S \partial r}\right) \\
& +2 H t^{2 H-1} \mu_{r}\left(\frac{t^{\alpha-1}}{\Gamma(\alpha)}\right)^{2 H} \frac{\partial C}{\partial r}+r S \frac{\partial C}{\partial S}-r C=0 .
\end{aligned}
$$

Let

$$
\begin{aligned}
& \tilde{\sigma}_{s}^{2}(t)=H t^{2 H-1} \sigma_{s}^{2}\left(\frac{t^{\alpha-1}}{\Gamma(\alpha)}\right)^{2 H}, \\
& \tilde{\sigma}_{r}^{2}(t)=H t^{2 H-1} \sigma_{r}^{2}\left(\frac{t^{\alpha-1}}{\Gamma(\alpha)}\right)^{2 H} .
\end{aligned}
$$


Then

$$
\begin{aligned}
& \frac{\partial C}{\partial t}+\widetilde{\sigma}_{s}^{2}(t) S_{t}^{2} \frac{\partial^{2} C}{\partial S_{t}^{2}}+\widetilde{\sigma}_{r}^{2}(t) \frac{\partial^{2} C}{\partial r^{2}}+2 \rho \widetilde{\sigma}_{r}(t) \widetilde{\sigma}_{s}(t) \frac{\partial^{2} C}{\partial S \partial r} \\
& +2 H t^{2 H-1} \mu_{r}\left(\frac{t^{\alpha-1}}{\Gamma(\alpha)}\right)^{2 H} \frac{\partial C}{\partial r}+r S \frac{\partial C}{\partial S}-r C=0,
\end{aligned}
$$

proof is completed.

From Theorem (3.1), we can get the following corollaries

Corollary 3.1. If $\rho=0$ and $r(t)$ be a constant, then the European call option $C=C(S, r, T)$ satisfies

$$
\frac{\partial C}{\partial t}+H t^{2 H-1} \sigma_{s}^{2} S_{t}^{2}\left(\frac{t^{\alpha-1}}{\Gamma(\alpha)}\right)^{2 H} \frac{\partial^{2} C}{\partial S_{t}^{2}}+r S \frac{\partial C}{\partial S}-r C=0
$$

which is a fractional BS equation considered in [10].

Corollary 3.2. When $\alpha \uparrow 1$, we obtain

$$
\begin{aligned}
& \frac{\partial C}{\partial t}+H t^{2 H-1} \sigma_{s}^{2} S_{t}^{2} \frac{\partial^{2} C}{\partial S_{t}^{2}}+H t^{2 H-1} \sigma_{r}^{2} \frac{\partial^{2} C}{\partial r^{2}}+2 H t^{2 H-1} \rho \sigma_{r} \sigma_{s} \frac{\partial^{2} C}{\partial S \partial r} \\
& +2 H t^{2 H-1} \mu_{r} \frac{\partial C}{\partial r}+r S \frac{\partial C}{\partial S}-r C=0,
\end{aligned}
$$

Further, if $\rho=0, H=\frac{1}{2}$, and $r(t)$ be a constant, from Equation (3.12) we have the celebrated $B S$ equation

$$
\frac{\partial C}{\partial t}+\frac{1}{2} \sigma_{s}^{2} S_{t}^{2} \frac{\partial^{2} C}{\partial S_{t}^{2}}+r S \frac{\partial C}{\partial S}-r C=0
$$

\section{Pricing Formula Under SUbdiffusive FraCtionalBlack-Scholes MODEL}

In this section, we propose an explicit formula for European call option when its value satisfies the partial differential equation (3.1) with boundary condition $C(S, r, T)=\left(S_{T}-K\right)^{+}$. Then, we can get

Theorem 4.1. Let $r(t)$ satisfies Equation (1.3) and $S(t)$ satisfies Equation (1.1), then the price of European call and put options with strike price $K$ and maturity $T$ are given by

$$
\begin{aligned}
& C(S, r, t)=S \phi\left(d_{1}\right)-K P(r, t, T) \phi\left(d_{2}\right), \\
& P(S, r, t)=K P(r, t, T) \phi\left(-d_{2}\right)-\phi\left(-d_{1}\right) .
\end{aligned}
$$

where

$$
\begin{aligned}
d_{1} & =\frac{\ln \frac{S}{K}-\ln P(r, t, T)+\frac{H}{(\Gamma(\alpha))^{2 H}} \int_{t}^{T} \widehat{\sigma}^{2}(s) s^{(\alpha-1) 2 H+2 H-1} d s}{\sqrt{\frac{2 H}{(\Gamma(\alpha))^{2 H}} \int_{t}^{T} \widehat{\sigma}^{2}(s) s^{(\alpha-1) 2 H+2 H-1} d s}}, \\
d_{2} & =d_{1}-\sqrt{\frac{2 H}{(\Gamma(\alpha))^{2 H}} \int_{t}^{T} \widehat{\sigma}^{2}(s) s^{(\alpha-1) 2 H+2 H-1} d s}, \\
\widehat{\sigma}^{2}(t) & =\sigma_{s}^{2}+2 \rho \sigma_{r} \sigma_{s}(T-t)+\sigma_{r}^{2}(T-t)^{2} .
\end{aligned}
$$


$P(r, t, T)$ is given by Equation (2.16) and $\phi($.$) is the cumulative normal distribution$ function.

\section{Proof:}

Consider the partial differential equation (3.1) of the European call option with boundary condition $C(S, r, T)=\left(S_{T}-K\right)^{+}$

$$
\begin{aligned}
& \frac{\partial C}{\partial t}+\widetilde{\sigma}_{s}^{2}(t) S_{t}^{2} \frac{\partial^{2} C}{\partial S_{t}^{2}}+\widetilde{\sigma}_{r}^{2}(t) \frac{\partial^{2} C}{\partial r^{2}}+2 \rho \widetilde{\sigma}_{r}(t) \widetilde{\sigma}_{s}(t) \frac{\partial^{2} C}{\partial S \partial r} \\
& +2 H t^{2 H-1} \mu_{r}\left(\frac{t^{\alpha-1}}{\Gamma(\alpha)}\right)^{2 H} \frac{\partial C}{\partial r}+r S \frac{\partial C}{\partial S}-r C=0 .
\end{aligned}
$$

Denote

$$
z=\frac{S}{P(r, t, T)}, \quad \Theta(z, t)=\frac{C(S, r, t)}{P(r, t, T)},
$$

therefore by computing, we get

$$
\begin{aligned}
\frac{\partial C}{\partial t} & =\Theta \frac{\partial P}{\partial t}+P \frac{\partial \Theta}{\partial t}-z \frac{\partial \Theta}{\partial z} \frac{\partial P}{\partial t}, \\
\frac{\partial C}{\partial r} & =\Theta \frac{\partial P}{\partial r}-z \frac{\partial \Theta}{\partial z} \frac{\partial P}{\partial r}, \\
\frac{\partial C}{\partial S} & =\frac{\partial \Theta}{\partial z}, \\
\frac{\partial^{2} C}{\partial r^{2}} & =\Theta \frac{\partial^{2} P}{\partial r^{2}}-z \frac{\partial \Theta}{\partial z} \frac{\partial^{2} P}{\partial r^{2}}+\frac{z^{2}}{P} \frac{\partial^{2} \Theta}{\partial z^{2}}\left(\frac{\partial P}{\partial r}\right)^{2}, \\
\frac{\partial^{2} C}{\partial r \partial S} & =-\frac{z}{P} \frac{\partial^{2} \Theta}{\partial z^{2}} \frac{\partial P}{\partial r}, \\
\frac{\partial^{2} C}{\partial S^{2}} & =\frac{1}{P} \frac{\partial^{2} \Theta}{\partial z^{2}} .
\end{aligned}
$$

Inserting Equation (4.8) into Equation (4.6)

$$
\begin{aligned}
\frac{\partial \Theta}{\partial t} & +\frac{\partial^{2} \Theta}{\partial z^{2}}\left[\widetilde{\sigma}_{s}^{2}(t) \frac{S^{2}}{P^{2}}+2 \rho z^{2} \widetilde{\sigma}_{r}(t) \widetilde{\sigma}_{s}(t) \frac{1}{P} \frac{\partial P}{\partial r}+\widetilde{\sigma}_{r}^{2}(t) z^{2}\left(\frac{1}{P} \frac{\partial P}{\partial r}\right)^{2}\right] \\
& -\frac{z}{P}\left[\frac{\partial P}{\partial t}+\widetilde{\sigma}_{r}^{2}(t) \frac{\partial^{2} P}{\partial r^{2}}+2 H t^{2 H-1} \mu_{r}\left(\frac{t^{\alpha-1}}{\Gamma(\alpha)}\right)^{2 H} \frac{\partial P}{\partial r}-r \frac{S}{z}\right] \\
& +\frac{\Theta}{P}\left[\frac{\partial P}{\partial t}+\widetilde{\sigma}_{r}^{2}(t) \frac{\partial^{2} P}{\partial r^{2}}+2 H t^{2 H-1} \mu_{r}\left(\frac{t^{\alpha-1}}{\Gamma(\alpha)}\right)^{2 H} \frac{\partial P}{\partial r}-r P\right]=0
\end{aligned}
$$

From Equation (2.8), we can obtain

$$
\frac{\partial \Theta}{\partial t}+\bar{\sigma}^{2}(t) z^{2} \frac{\partial^{2} \Theta}{\partial z^{2}}=0,
$$

with boundary condition $\Theta(z, T)=(z-K)^{+}$, 
where

$$
\bar{\sigma}^{2}(t)=\widetilde{\sigma}_{s}^{2}(t)+2 \rho \widetilde{\sigma}_{r}(t) \widetilde{\sigma}_{s}(t)(T-t)+\widetilde{\sigma}_{r}(t)^{2}(T-t)^{2} .
$$

The solution of partial differential Equation (4.10) with boundary condition $\Theta(z, T)=(z-K)^{+}$, is given by

$$
\Theta(z, t)=z \phi\left(\widehat{d}_{1}\right)-K \phi\left(\widehat{d}_{2}\right)
$$

here

$$
\begin{aligned}
& \widehat{d}_{1}=\frac{\ln \frac{z}{K}+\int_{t}^{T} \bar{\sigma}^{2}(s) d s}{\sqrt{2 \int_{t}^{T} \widehat{\sigma}^{2}(s) d s}}, \\
& \widehat{d}_{2}=\widehat{d}_{1}-\sqrt{2 \int_{t}^{T} \bar{\sigma}^{2}(s) d s .}
\end{aligned}
$$

Thus, from Equations (4.7) and (4.12)-(4.14) we obtain

$$
C(S, r, t)=S \phi\left(d_{1}\right)-K P(r, t, T) \phi\left(d_{2}\right),
$$

where

$$
\begin{aligned}
d_{1} & =\frac{\ln \frac{S}{K}-\ln P(r, t, T)+\frac{H}{(\Gamma(\alpha))^{2 H}} \int_{t}^{T} \widehat{\sigma}^{2}(s) s^{(\alpha-1) 2 H+2 H-1} d s}{\sqrt{\frac{2 H}{(\Gamma(\alpha))^{2 H}} \int_{t}^{T} \widehat{\sigma}^{2}(s) s^{(\alpha-1) 2 H+2 H-1} d s}}, \\
d_{2} & =d_{1}-\sqrt{\frac{2 H}{(\Gamma(\alpha))^{2 H}} \int_{t}^{T} \widehat{\sigma}^{2}(s) s^{(\alpha-1) 2 H+2 H-1} d s}
\end{aligned}
$$

Letting $\alpha \uparrow 1$, from Theorem 4.1, we obtain

Corollary 4.1. The price of European call and put options with strike price $K$ and maturity $T$ are given by

$$
\begin{aligned}
& C(S, r, T)=S \phi\left(d_{1}\right)-K P(r, t, T) \phi\left(d_{2}\right), \\
& P(S, r, T)=K P(r, t, T) \phi\left(-d_{2}\right)-S \phi\left(-d_{1}\right) .
\end{aligned}
$$

where

$$
\begin{aligned}
d_{1}= & \frac{\ln \frac{S}{K}-\ln P(r, t, T)+H \int_{t}^{T} \widehat{\sigma}^{2}(s) s^{2 H-1} d s}{\sqrt{2 H \int_{t}^{T} \widehat{\sigma}^{2}(s) s^{2 H-1} d s}}, \\
d_{2}= & d_{1}-\sqrt{2 H \int_{t}^{T} \widehat{\sigma}^{2}(s) s^{2 H-1} d s} \\
\widehat{\sigma}^{2}(t)= & \sigma_{s}^{2}+2 \rho \sigma_{r} \sigma_{s}(T-t)+\sigma_{r}^{2}(T-t)^{2}, \\
P(r, t, T)= & \exp \left\{-r \tau+H \sigma_{r}^{2} \int_{0}^{\tau}(T-s)^{2 H-1} s^{2} d s\right. \\
& \left.-2 H \mu_{r} \int_{0}^{\tau}(T-s)^{2 H-1} s d s\right\}, \tau=T-t .
\end{aligned}
$$


More specifically, if $H=\frac{1}{2}$, we have

$$
\begin{aligned}
d_{1} & =\frac{\ln \frac{S}{K}-\ln P(r, t, T)+\frac{1}{2} \varphi(t, T)}{\sqrt{\varphi(t, T)}}, \\
d_{2} & =d_{1}-\sqrt{\varphi(t, T)}, \\
\varphi(t, T) & =\sigma_{s}^{2}(T-t)+\rho \sigma_{r} \sigma_{s}(T-t)^{2}+\frac{1}{3} \sigma_{r}^{2}(T-t)^{3}, \\
P(r, t, T) & =\exp \left\{-r(T-t)-\frac{1}{2} \mu_{r}(T-t)^{2}+\frac{1}{6} \sigma_{r}^{2}(T-t)^{3}\right\} .
\end{aligned}
$$

which is consistent with result in [3].

Letting $H=\frac{1}{2}$, from Theorem 4.1, we can get

Corollary 4.2. The price of European call and put options with strike price $K$ and maturity $T$ are given by

$$
\begin{aligned}
& C(S, r, T)=S \phi\left(d_{1}\right)-K P(r, t, T) \phi\left(d_{2}\right), \\
& P(S, r, T)=K P(r, t, T) \phi\left(-d_{2}\right)-\phi\left(-d_{1}\right) .
\end{aligned}
$$

where

$$
\begin{aligned}
d_{1}= & \frac{\ln \frac{S}{K}-\ln P(r, t, T)+\frac{1}{2 \Gamma(\alpha)} \int_{t}^{T} \widehat{\sigma}^{2}(s) s^{\alpha-1} d s}{\sqrt{\frac{1}{\Gamma(\alpha)} \int_{t}^{T} \widehat{\sigma}^{2}(s) s^{\alpha-1} d s}}, \\
d_{2}= & d_{1}-\sqrt{\frac{1}{\Gamma(\alpha)} \int_{t}^{T} \widehat{\sigma}^{2}(s) s^{\alpha-1} d s} \\
\widehat{\sigma}^{2}(t)= & \sigma_{s}^{2}+2 \rho \sigma_{r} \sigma_{s}(T-t)+\sigma_{r}^{2}(T-t)^{2}, \\
P(r, t, T)= & \exp \left\{-r \tau+\frac{\sigma_{r}^{2}}{2 \Gamma(\alpha)} \int_{0}^{\tau}(T-s)^{\alpha-1} s^{2} d s\right. \\
& \left.-\frac{\mu_{r}}{(\Gamma(\alpha)} \int_{0}^{\tau}(T-s)^{\alpha-1} s d s\right\} .
\end{aligned}
$$

Specially, If $\rho=0$, from Equations (4.28)-(4.33), we have

$$
\begin{aligned}
d_{1}= & \frac{\ln \frac{S}{K}-\ln P(r, t, T)+\frac{1}{2 \Gamma(\alpha)} \int_{t}^{T} \widehat{\sigma}^{2}(s) s^{\alpha-1} d s}{\sqrt{\frac{1}{\Gamma(\alpha)} \int_{t}^{T} \widehat{\sigma}^{2}(s) s^{\alpha-1} d s}}, \\
d_{2}= & d_{1}-\sqrt{\frac{1}{\Gamma(\alpha)} \int_{t}^{T} \widehat{\sigma}^{2}(s) s^{\alpha-1} d s,} \\
\widehat{\sigma}^{2}(t)= & \sigma_{s}^{2}+\sigma_{r}^{2}(T-t)^{2}, \\
P(r, t, T)= & \exp \left\{-r \tau+\frac{1}{2} \frac{\sigma_{r}^{2}}{\Gamma(\alpha)} \int_{0}^{\tau}(T-s)^{\alpha-1} s^{2} d s\right. \\
& \left.-\frac{\mu_{r}}{\Gamma(\alpha)} \int_{0}^{\tau}(T-s)^{\alpha-1} s d s\right\} .
\end{aligned}
$$

which is similar with results mentioned in [6]. 


\section{Simulation Studies}

Let us first discuss about the implied volatility of the subdiffusive $F B S$ model, then we will show some simulation findings.

Corollary 5.1. If $t=0$, the value of European call option $\bar{C}(K, T)$ and put option $\bar{P}(K, T)$ can be written as

$$
\begin{aligned}
& \bar{C}(K, T)=S_{0} \phi\left(\bar{d}_{1}\right)-K P_{0} \phi\left(\bar{d}_{2}\right), \\
& \bar{P}(K, T)=K P_{0} \phi\left(-\bar{d}_{2}\right)-S_{0} \phi\left(-\bar{d}_{1}\right) .
\end{aligned}
$$

where

$$
\begin{aligned}
P_{0} & =\exp \left\{-r_{0} T+\frac{2 H T^{(\alpha-1) 2 H+2 H+1}}{(\Gamma(\alpha))^{2 H}((\alpha-1) 2 H+2 H)((\alpha-1) 2 H+2 H+1)}\right. \\
& \left.\times\left(\frac{\sigma_{r}^{2} T}{(\alpha-1) 2 H+2 H+2}-\mu_{r}\right)\right\}
\end{aligned}
$$

$(5.4) \bar{d}_{1}=\frac{\ln \frac{S_{0}}{K}+\bar{r} T+\frac{1}{2} \bar{\sigma}^{2} T}{\bar{\sigma} \sqrt{T}}$,

$(5.5) \bar{d}_{2}=\bar{d}_{1}-\bar{\sigma} \sqrt{T}$

$(5.6) \bar{r}=r_{0}+\frac{2 H T^{(\alpha-1) 2 H+2 H}}{(\Gamma(\alpha))^{2 H}((\alpha-1) 2 H+2 H)((\alpha-1) 2 H+2 H+1)}$

$$
\times\left(\mu_{r}-\frac{\sigma_{r}^{2} T}{(\alpha-1) 2 H+2 H+2}\right),
$$

$$
\bar{\sigma}^{2}=\frac{2 H T^{(\alpha-1) 2 H+2 H-1}}{(\Gamma(\alpha))^{2 H}((\alpha-1) 2 H+2 H)}\left(\sigma_{s}^{2}+\frac{\rho \sigma_{r} \sigma_{s} T}{(\alpha-1) 2 H+2 H+1}\right.
$$

$$
\left.+\frac{\sigma_{r}^{2} T^{2}}{((\alpha-1) 2 H+2 H+1)((\alpha-1) 2 H+2 H+2)}\right) \text {. }
$$

and $\phi($.$) is the cumulative normal distribution function.$

Table 1 indicates the theoretical prices from our $F B S$ and subdiffusive $F B S$ models and Merton and subdiffusive $B S$ models, where $S_{0}$ shows the stock price, $P_{M}$ presents the prices evaluated by the Merton model, $P_{S B S}$ denotes the price simulated by the subdiffusive $B S$ model, $P_{F B S}$ and $P_{S F B S}$ show the price obtained by the $F B S$ and subdiffusive $F B S$ models, respectively. 
TABLE 1. Results by different pricing models. Here, $\alpha=0.9, H=$ $0.6, K=3, \sigma_{r}=0.3, \sigma_{s}=0.4, \rho=0.4, \mu_{r}=0.5, r_{0}=0.3, T=$ $0.3, t=0$.

\begin{tabular}{|ccccccccc|}
\hline & \multicolumn{3}{c}{$T=0.2$} & & \multicolumn{3}{c|}{$T=1$} \\
\cline { 2 - 3 } \cline { 7 - 8 } S & $P_{M}$ & $P_{S B S}$ & $P_{F B S}$ & $P_{S F B S}$ & $P_{M}$ & $P_{S B S}$ & $P_{F B S}$ & $P_{S F B S}$ \\
\hline 2 & 0.0174 & 0.0334 & 0.0012 & 0.0036 & 1.8826 & 1.9129 & 1.7986 & 1.8347 \\
2.25 & 0.0638 & 0.0979 & 0.0122 & 0.0236 & 2.1326 & 2.1629 & 2.0486 & 2.0847 \\
2.5 & 0.1598 & 0.2126 & 0.0587 & 0.0859 & 2.3826 & 2.4129 & 2.2986 & 2.3347 \\
2.75 & 0.3094 & 0.3754 & 0.1687 & 0.2094 & 2.6326 & 2.6629 & 2.5486 & 2.5847 \\
3 & 0.5023 & 0.5752 & 0.3440 & 0.3900 & 2.8826 & 2.1929 & 2.7986 & 2.8347 \\
3.25 & 0.7235 & 0.7988 & 0.5630 & 0.6086 & 3.1326 & 3.1629 & 3.0486 & 3.0847 \\
3.5 & 0.9604 & 1.0360 & 0.8026 & 0.8466 & 3.3826 & 3.4129 & 3.2986 & 3.3347 \\
3.75 & 1.2094 & 1.2801 & 1.0498 & 1.0926 & 3.6326 & 3.6629 & 3.5486 & 3.5847 \\
4 & 1.4527 & 1.5275 & 1.2991 & 1.3414 & 3.8826 & 3.9129 & 3.7986 & 3.8347 \\
\hline
\end{tabular}

By comparing columns $P_{M}, P_{S B S}, P_{F B S}$ and $P_{S F B S}$ in Table 1, we conclude the call option prices obtained by four pricing models are close to each other in the both in-the-money and out-of-the-money cases with low and high maturities. Meanwhile, we can see that the prices given by the our $F B S$ and subdiffusive $F B S$ models are smaller than the prices given by the Merton and subdiffusive Merton models $[3,6]$.

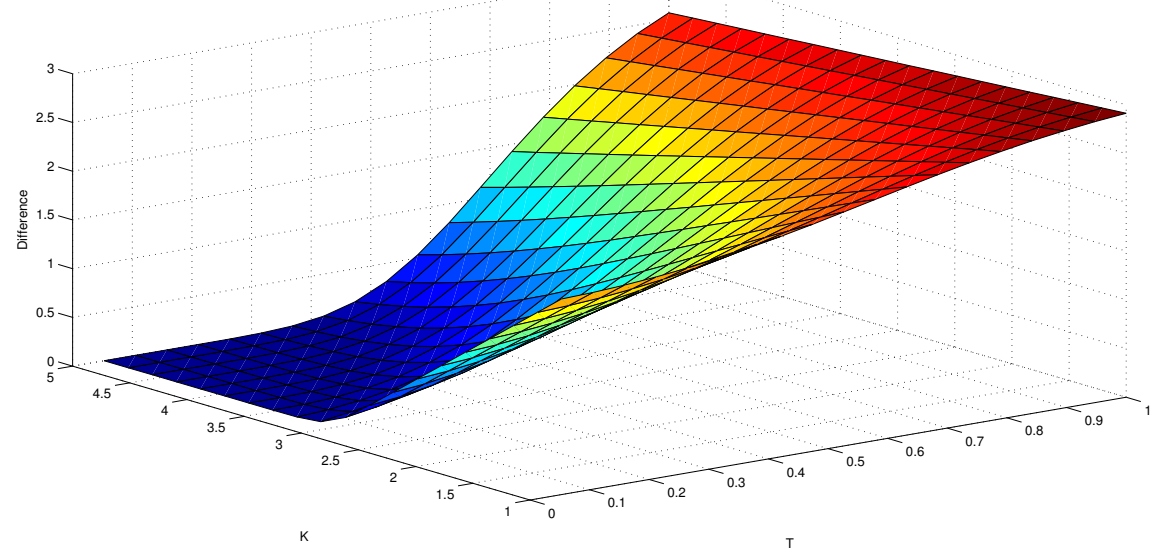

Figure 2. The European call option under subdiffusive $F B S$. Where $r_{0}=0.1, \alpha=0.9, H=0.8, \sigma_{r}=0.3, \sigma_{s}=0.4, S_{0}=3, \mu_{r}=$ $0.2, \rho=0.2$. 


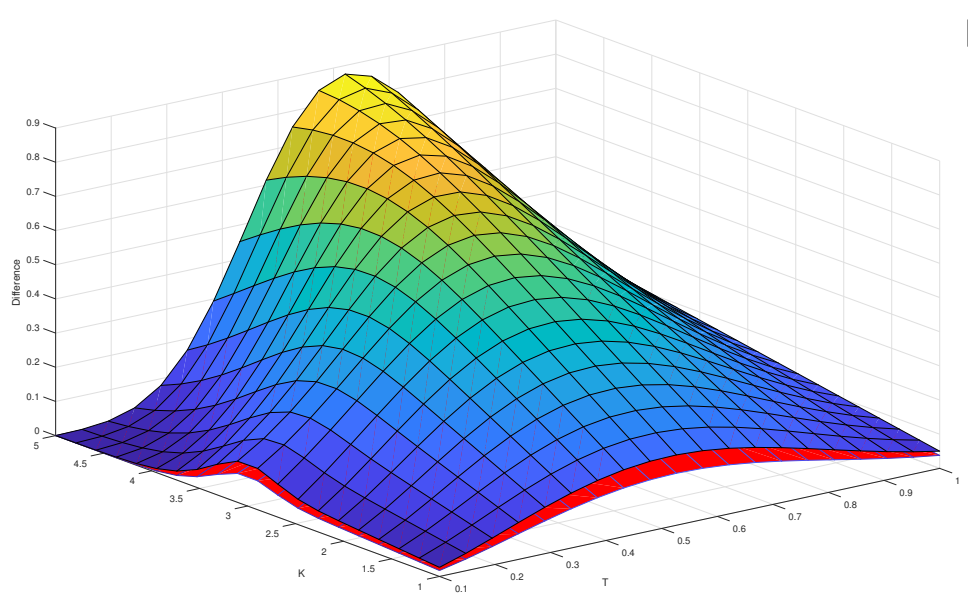

FiguRE 3. The difference between the price of the European call option under subdiffusive $F B S$, subdiffusive Merton and Merton models. Where $r_{0}=0.1, \alpha=0.9, H=0.8, \sigma_{r}=0.3, \sigma_{s}=0.4, S_{0}=$ $3, \mu_{r}=0.2, t=0, \rho=0.3$.

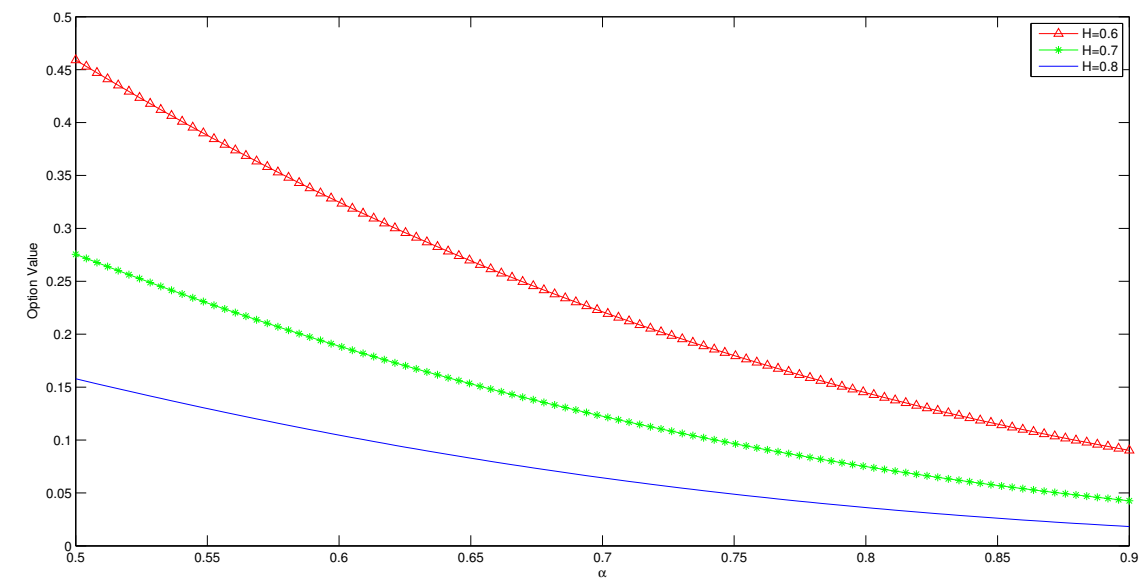

FiguRE 4. The European call option under subdiffusive $F B S$. Where $r_{0}=0.3, \sigma_{r}=0.1, \sigma_{s}=0.3, S_{0}=4, \mu_{r}=0.2, \rho=0.2, t=$ $0, T=0.2$.

From Equations (5.1)-(5.7), it is easy to see that $\bar{\sigma}$ and $\bar{r}$ is the implied volatility and implied short rate connected to the FBS model, respectively (See Fig 2, 3 and 4 ).

\section{Conclusion}

Most of prior pricing models have assumed the constant short rate during the life of an option. However, in real life the short rate is evolving randomly through time. For this purpose, we apply the subdiffusive mechanism to get better characteristic 
property of stock markets. We propose a pricing model for a zero-coupon bond when the short rate is governed by the subdiffusive fractional Black-Scholes model. Then, we exert these results to develop analytical valuation formulas for European option and corresponding fractional Black-Scholes equation.

We allow to referees to evaluate our manuscript.

\section{REFERENCES}

[1] F. Black And M. Scholes, The pricing of options and corporate liabilities, Journal of political economy, 81 (1973), pp. 637-654.

[2] P. Cheridito, Arbitrage in fractional brownian motion models, Finance and Stochastics, 7 (2003), pp. 533-553.

[3] Z. CUI AND D. MCLeish, Comment on "option pricing under the merton model of the short rate" by kung and lee [math. comput. simul. 80 (2009) 378-386], Mathematics and Computers in Simulation, 81 (2010), pp. 1-4.

[4] I. Eliazar and J. Klafter, Spatial gliding, temporal trapping, and anomalous transport, Physica D: Nonlinear Phenomena, 187 (2004), pp. 30-50.

[5] H. Gu, J.-R. LIANG, AND Y.-X. Zhang, Time-changed geometric fractional brownian motion and option pricing with transaction costs, Physica A: Statistical Mechanics and its Applications, 391 (2012), pp. 3971-3977.

[6] Z. Guo, Option pricing under the merton model of the short rate in subdiffusive brownian motion regime, Journal of Statistical Computation and Simulation, 87 (2017), pp. 519-529.

[7] M. HAhn, K. KoBAYASHI, AND S. UMAROv, Fokker-planck-kolmogorov equations associated with time-changed fractional brownian motion, Proceedings of the American mathematical Society, 139 (2011), pp. 691-705.

[8] J. Janczura And A. WyŁomańska, Subdynamics of financial data from fractional fokkerplanck equation, Acta Physica Polonica B, 40 (2009), pp. 1341-1351.

[9] J. J. Kung And L.-S. LeE, Option pricing under the merton model of the short rate, Mathematics and Computers in Simulation, 80 (2009), pp. 378-386.

[10] J.-R. Liang, J. WANG, L.-J. Lu, H. GU, W.-Y. QIU, AND F.-Y. REN, Fractional fokkerplanck equation and black-scholes formula in composite-diffusive regime, Journal of Statistical Physics, 146 (2012), pp. 205-216.

[11] M. Magdziarz, Black-scholes formula in subdiffusive regime, Journal of Statistical Physics, 136 (2009), pp. 553-564.

[12] R. Metzler, E. Barkai, and J. Klafter, Anomalous diffusion and relaxation close to thermal equilibrium: A fractional Fokker-Planck equation approach, Physical Review Letters, 82 (1999), p. 3563.

[13] C. NeCulA, Option pricing in a fractional brownian motion environment, Academy of Economic Studies Bucharest, Romania, Preprint, Academy of Economic Studies, Bucharest, (2002).

[14] L. C. G. Rogers, Arbitrage with fractional brownian motion, Mathematical Finance, 7 (1997), pp. 95-105.

[15] I. M. Sokolov, Solutions of a class of non-Markovian Fokker-Planck equations, Physical Review E, 66 (2002), p. 041101.

[16] T. Sottinen And E. Valkeila, On arbitrage and replication in the fractional black-scholes pricing model, Statistics \& Decisions/International mathematical Journal for stochastic methods and models, 21 (2003), pp. 93-108.

[17] J. WAng, J.-R. LiAng, L.-J. Lv, W.-Y. QiU, AND F.-Y. REN, Continuous time black-scholes equation with transaction costs in subdiffusive fractional brownian motion regime, Physica A: Statistical Mechanics and its Applications, 391 (2012), pp. 750-759. 